\title{
Efficacy and safety of parecoxib sodium for acute postoperative pain: A meta-analysis
}

\author{
WEI WEI ${ }^{1}$, TIANYUN ZHAO ${ }^{2}$ and YUANTAO LI $^{2}$ \\ ${ }^{1}$ Department of Anesthesiology, Meizhou People's Hospital, Meizhou, Guangdong 514031; \\ ${ }^{2}$ Department of Anesthesiology, Shenzhen Maternity and Child Healthcare Hospital, \\ Southern Medical University, Shenzhen, Guangdong 518028, P.R. China
}

Received February 1, 2013; Accepted April 22, 2013

DOI: $10.3892 /$ etm.2013.1172

\begin{abstract}
This meta-analysis was performed to evaluate the efficacy and safety of parecoxib sodium for acute postoperative pain. PubMed, Cochrane Central Register of Controlled Trials, EBSCO, Springer, Ovid and Chinese National Knowledge Infrastructure (CNKI) databases were searched from January 1999 to January 2013 to comprehensively collect randomized controlled trials (RCTs) of parecoxib sodium for acute postoperative pain. The methodological quality of the included RCTs were assessed and the data were extracted by two reviewers independently according to the Cochrane Handbook. Efficacies and safety (respiratory depression, pruritus, fever, headache, and nausea and vomiting) were pooled using meta-analysis performed by Review Manager 5.1 software. Relative risk (RR) and 95\% confidence interval (CI) were calculated in a fixed-effects model. Seven RCTs involving 1,939 patients met the inclusion criteria. The results of the meta-analysis revealed that the rate of 'effective' treatment as described by the patients' global evaluation of study medication (PGESM) was higher in the patient-controlled analgesia (PCA) combined with parecoxib sodium group 24,48 , and $72 \mathrm{~h}$ after the initial intravenous dose of $40 \mathrm{mg}$ parecoxib compared with that in the control group [PCA alone; $\mathrm{RR}=1.41,95 \% \mathrm{CI}$ (1.13-1.75); RR=1.25, 95\% CI (1.15-1.35); and $\mathrm{RR}=1.30,95 \% \mathrm{CI}$ (1.21-1.40), respectively]. The rate of 'ineffective' treatment in the PCA combined with parecoxib sodium group was lower compared with that of the control group $[\mathrm{RR}=0.43,95 \% \mathrm{CI}$ (0.26-0.72); RR=0.44, 95\% CI (0.34-0.57); and $\mathrm{RR}=0.33$, 95\% CI (0.23-0.48), respectively]. Combination of PCA with parecoxib sodium reduced the incidence of postoperative fever $[\mathrm{RR}=0.34,95 \% \mathrm{CI}(0.22-0.53)]$, as well as nausea and vomiting $[\mathrm{RR}=0.69,95 \% \mathrm{CI}(0.57-0.83)]$; however, it did not significantly reduce respiratory depression $[R R=0.84,95 \%$
\end{abstract}

Correspondence to: Dr Yuantao Li, Department of Anesthesiology, Shenzhen Maternity and Child Healthcare Hospital, Southern Medical University, Shenzhen, Guangdong 518028, P.R. China E-mail: liyuantao@126.com

Key words: parecoxib sodium, postoperative pain, meta-analysis
CI (0.38-1.83)], pruritus [ $\mathrm{RR}=0.91,95 \% \mathrm{CI}(0.54-1.52)]$ or headache $[\mathrm{RR}=0.77,95 \% \mathrm{CI}(0.47-1.28)]$. The combination of PCA with parecoxib sodium successively injected for $<3$ days significantly increases the scores of PGESM and reduces the incidence of adverse effects and postoperative complications.

\section{Introduction}

Acute pain occurs as a result of tissue damage, often accidentally due to an injury or surgery. Acute postoperative pain is a manifestation of inflammation due to tissue injury. The management of postoperative pain and inflammation is a critical component of patient care (1).

Non-steroidal anti-inflammatory drugs (NSAIDs) are commonly used in the management of post-operative pain. NSAIDs inhibit cyclooxygenase (COX) enzymes, which are involved in the synthesis of prostaglandins and thereby reduce pain and inflammation. The inhibition of COX is the principal mechanism for the efficacy and the toxicity of NSAIDs (2) and it has been demonstrated that COX exists as at least two isoenzymes, COX-1 and COX-2 (3). Traditional NSAIDs non-specifically inhibit COX-1 and COX-2, whereas specific COX-2 inhibitors only affect the activity of COX-2. The major reason for development of specific COX-2 inhibitors was the maintenance of the anti-inflammatory and analgesic effects without altering the homeostatic functions of COX-1 (4). To represent an attractive alternative for patients requiring NSAIDs perioperatively, the selective COX-2 inhibitors, besides their improved side-effect profile, should have an equipotent analgesic efficacy relative to traditional NSAIDs.

Furthermore, oral NSAIDs are used post-operatively; however, when patients are unable to tolerate oral medications or require a faster onset of analgesia, parenteral administration may be preferred. Parecoxib is a COX-2 selective inhibitor, which may be administered as an intravenous or intramuscular injection for the short-term management of postoperative pain. It is a prodrug (the parent drug is inactive) that is rapidly hydrolysed in vivo to its active form, valdecoxib (5). Clinical trials have indicated that parecoxib is effective in treating postoperative pain resulting from oral surgery, orthopedic surgery and abdominal hysterectomy pain. Other studies have demonstrated no significant effects on platelet function or upper gastrointestinal mucosa (6-9). As a result, parecoxib 
sodium has been approved in European countries for the treatment of postoperative pain.

The combination of PCA and the selective COX-2 inhibitor parecoxib has reportedly been used for acute postoperative pain for years in European countries; however, the efficacy and safety of the combination has not yet been investigated. Therefore, to investigate the efficacy and safety profile of the combination of PCA and parecoxib for postoperative analgesic effects, we conducted a meta-analysis of randomized controlled trials (RCTs).

\section{Materials and methods}

Search sources and strategy. The search strategy was produced according to working handbook version 4.2.7 from the Cochrane collaboration (10). Studies were identified by extensively searching PubMed, Cochrane Central Register of Controlled Trials, EBSCO, Springer, Ovid and Chinese National Knowledge Infrastructure (CNKI) databases from January 1999 to January 2013. In addition, a manual search of abstracts from selected conferences was conducted, as well as a search by hand of the bibliographies of all relevant trials. The following search criteria were used: 'parecoxib sodium', 'cyclooxygenase-2 inhibitor' and 'RCTs'. The language of the studies was not restricted to English.

Study selection. Two reviewers independently conducted the literature search and extraction of relevant articles. The title and abstract of potentially relevant studies were screened for appropriateness before retrieval of the full articles. Any disagreement concerning study selection or data extraction was resolved by consensus with the third reviewer. For meta-analysis, all studies had to meet the following inclusion criteria: i) a study described as an RCT; ii) patients with no statistically significant differences in baseline characteristics; iii) intervention: a) treatment group, PCA combined with parecoxib sodium (successively injected for $<3$ days) intravenously at 40+20/40 mg bid; b) control group, same volume of saline; and iv) outcome variables: a) according to patients' global evaluation of study medication (PGESM), pain relief 24, 48 and $72 \mathrm{~h}$ after the initial intravenous dose of $40 \mathrm{mg}$ parecoxib was assessed on a four-point scale ( 0 , none; 1 , a little or some; 2, a lot; and 3, complete; scores 1 and 2 were defined as 'ineffective' and 3 and 4 were defined as 'effective'); b) adverse reactions of opioids, including respiratory depression, pruritus, fever, headache, nausea and vomiting.

The exclusion criteria were as follows: i) a single injection of parecoxib sodium before PCA; and ii) PCA not combined with parecoxib sodium following surgery.

Data extraction and assessment of study quality. Two of the authors independently extracted data from the trials that met the inclusion criteria. Authors were contacted for missing data when necessary. From each study, the following information was extracted: author, year of publication, sample size and intervention measures.

Quality assessment of the RCTs included in the meta-analysis was independently performed by the same reviewers according to the Cochrane Handbook 5.0.1 and Juni et al $(11,12)$. Jadad grade was evaluated using the following items: i) was the study a randomized trial; ii) was

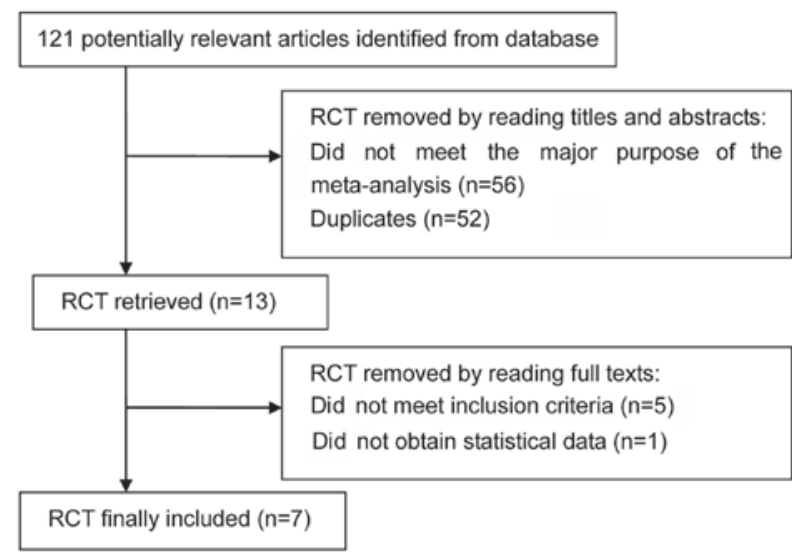

Figure 1. Study selection process. RCT, randomized controlled trial.

the randomization scheme described and appropriate; iii) was the study described as double-blinded; iv) was the method of double-blinding appropriate; v) was there a description of allocation concealment; vi) was there a description of dropouts and withdrawals; and (vii) did the patients have statistically significant differences in baseline characteristics. Each author rated the quality of the trials using Jadad grade (maximum grade, A; minimum grade, $C$; grade $\geq B$, good quality).

Statistical analysis. Data were analyzed using Review Manager 5.1 (provided by The Nordic Cochrane Centre, The Cochrane Collaboration). Included articles were pooled and weighted (13). Relative risk (RR) and 95\% confidence interval (CI) were calculated in a random-effects model or in a fixed-effects model. Heterogeneity was assessed by $\chi^{2}$ test and the quantity of heterogeneity was measured with $\mathrm{I}^{2}$ statistic. If heterogeneity $\left(\mathrm{P}<0.01\right.$ or $\left.\mathrm{I}^{2}>50 \%\right)$ was identified among the trials, a random-effects model was selected, otherwise a fixed-effects model was selected. If heterogeneity was evident $\left(\mathrm{I}^{2}>70 \%\right)$, the inferior quality study was eliminated for analysis.

\section{Results}

Study characteristics. There were 121 articles relevant to the search terms and seven articles (14-20) involving 1,939 patients (treatment group, 1,207 patients; control group, 732 patients) were included in this meta-analysis. The flow chart for the selection of RCTs is shown in Fig. 1. The characteristics of the included trials are shown in Table I.

Methodological quality assessment. The quality assessment of included RCTs is presented in Table I. All the trials included in this meta-analysis clarified adequate randomization procedures, used double-blinding and reported numbers of dropouts/withdrawals during the treatment; however, no study reported allocation concealment clearly. According to the Jadad score, all studies were eventually assessed to be good in terms of methodology with Jadad score B.

\section{Comparisons of effectiveness}

Patient global evaluation $24 \mathrm{~h}$ after the initial dose of parecoxib. Two studies $(17,20)(n=166)$ provided specific data 


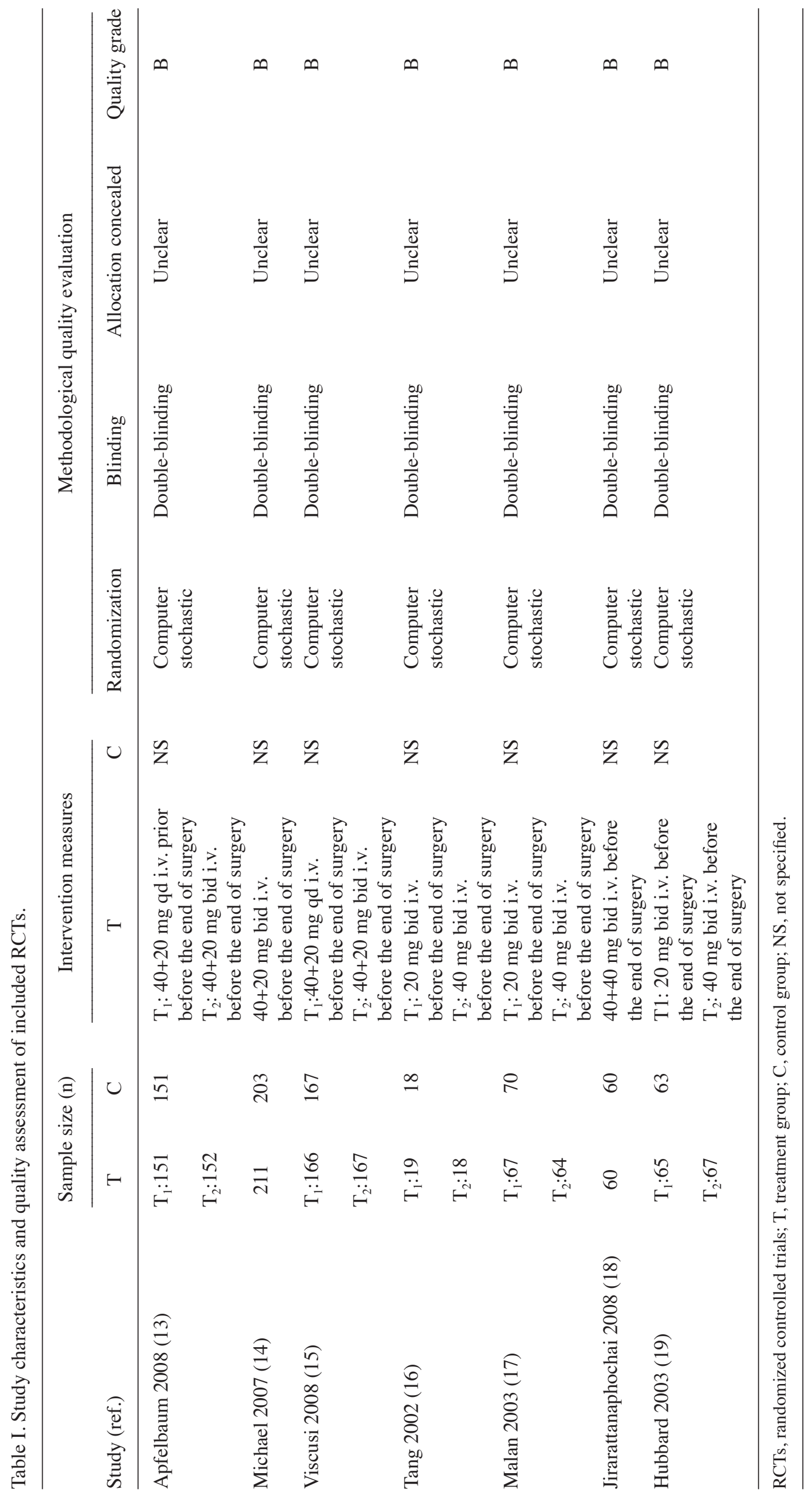




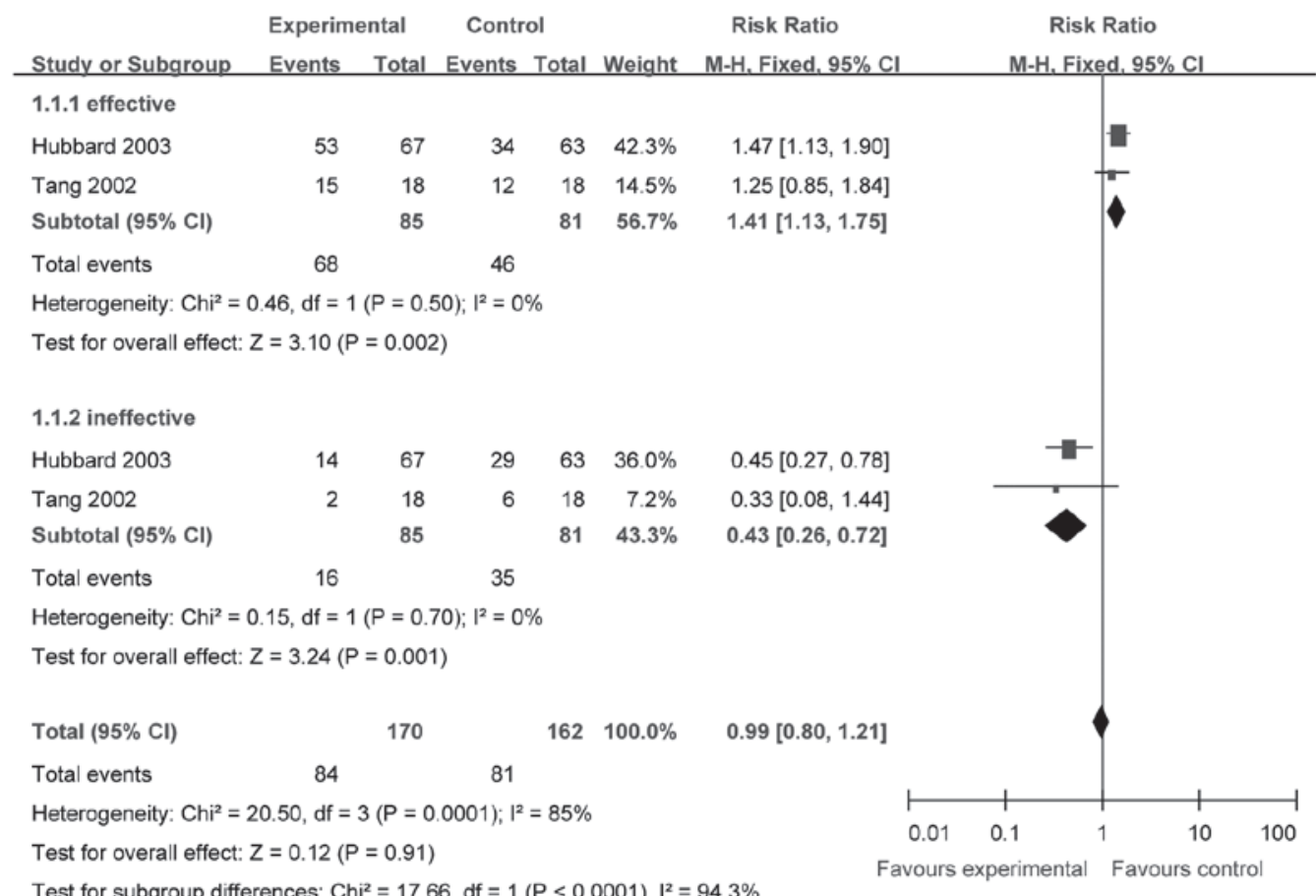

Figure 2. Meta-analysis of patients' global evaluation of study medication of PCA combined with parecoxib sodium and PCA alone at $24 \mathrm{~h}$ after surgery. PCA, patient-controlled analgesia.

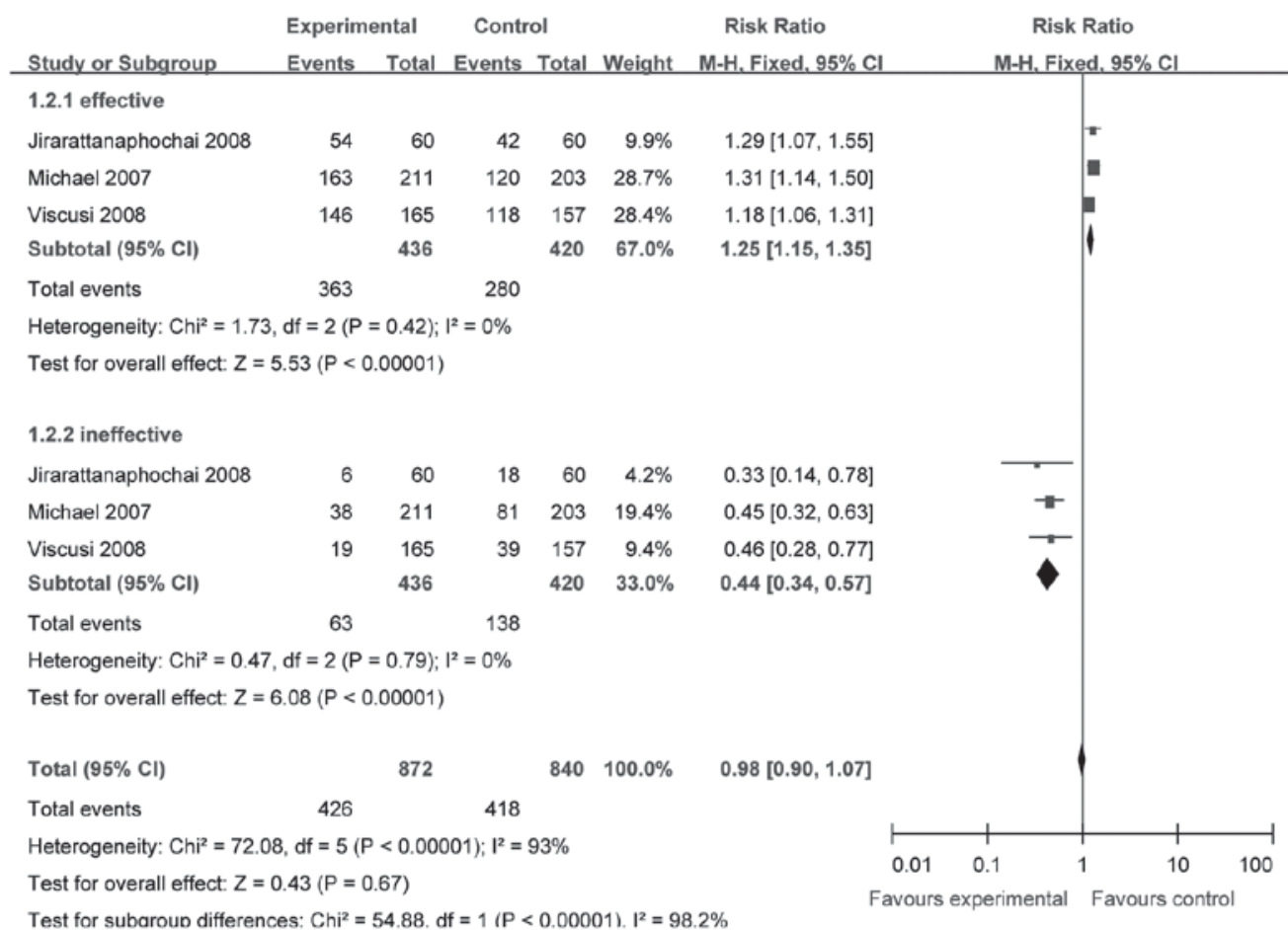

Figure 3. Meta-analysis of patients' global evaluation of study medication of PCA combined with parecoxib sodium and PCA alone at $48 \mathrm{~h}$ after surgery. PCA, patient-controlled analgesia.

for analysis of PGESM at $24 \mathrm{~h}$ after surgery. We selected the fixed-effect model to perform the meta-analysis since there were no significant heterogeneities (effective, $\chi^{2}=0.46, \mathrm{P}=0.50$, $\mathrm{I}^{2}=0 \%$; ineffective, $\chi^{2}=0.15, \mathrm{P}=0.70, \mathrm{I}^{2}=0 \%$ ). The incidence of 'effective' and 'ineffective' results in the analgesic effect evaluation was significantly different between the two groups
[RR $=1.41,95 \% \mathrm{CI}(1.13-1.75), \mathrm{P}=0.002$; and $\mathrm{RR}=0.43,95 \% \mathrm{CI}$ (0.26-0.72), $\mathrm{P}=001$, respectively; Fig. 2].

Patient global evaluation $48 \mathrm{~h}$ after the initial dose of parecoxib. Three studies $(15,16,19)(\mathrm{n}=868)$ provided specific data for analysis of PGESM at $48 \mathrm{~h}$ after surgery. We selected the fixed-effect model to perform the meta-analysis since there 


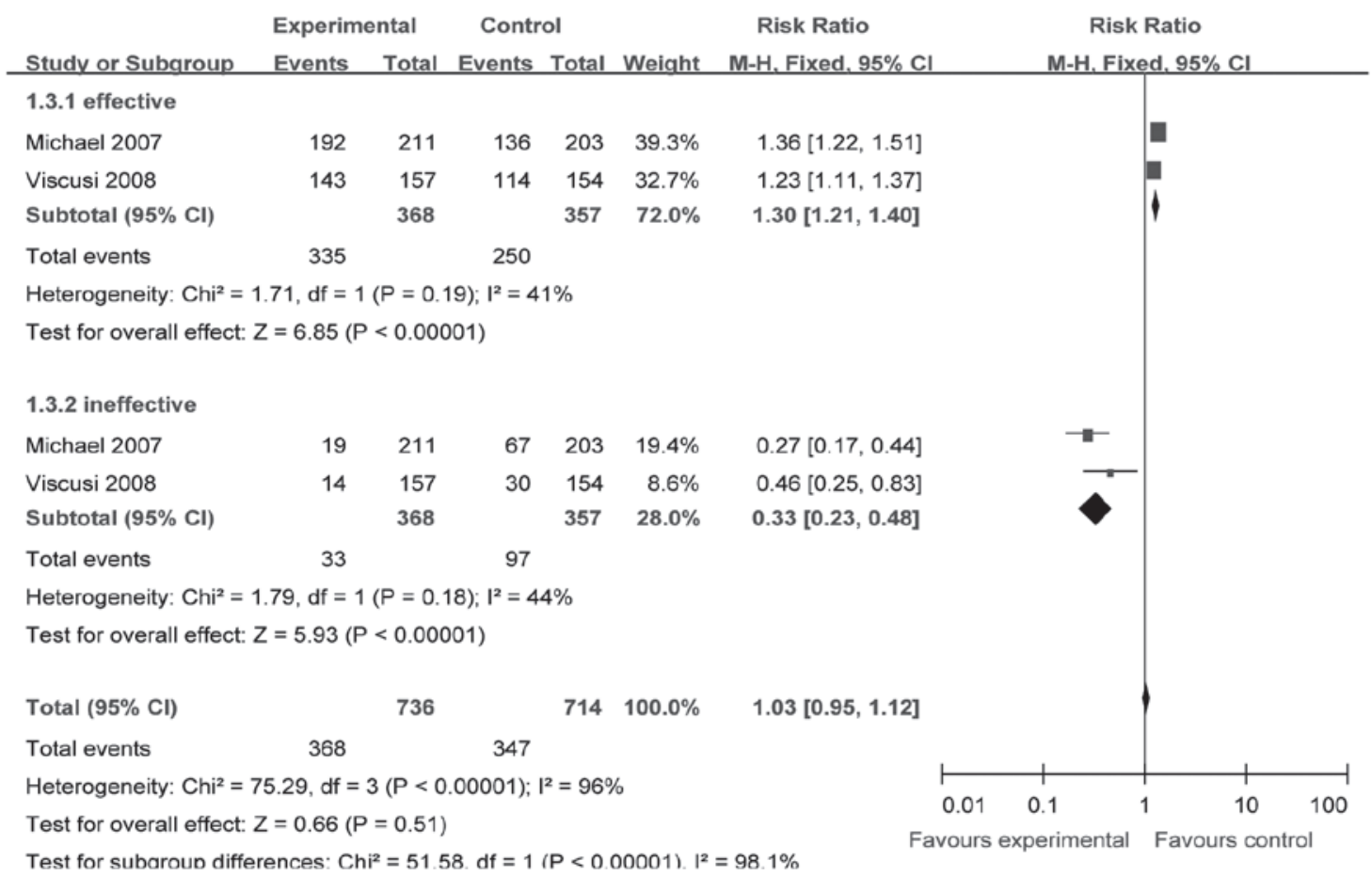

Figure 4. Meta-analysis of patients' global evaluation of study medication of PCA combined with parecoxib sodium and PCA alone at $72 \mathrm{~h}$ after surgery. PCA, patient-controlled analgesia.

were no significant heterogeneities (effective, $\chi^{2}=1.73, \mathrm{P}=0.42$, $\mathrm{I}^{2}=0 \%$; ineffective $\left.\chi^{2}=0.47, \mathrm{P}=0.79, \mathrm{I}^{2}=0 \%\right)$. The incidence of 'effective' and 'ineffective' results in the analgesic effect evaluation was significantly different between the two groups $[\mathrm{RR}=1.25,95 \% \mathrm{CI}(1.15-1.35), \mathrm{P}<0.00001$; and $\mathrm{RR}=0.44,95 \%$ CI (0.34-0.57), $\mathrm{P}<0.00001$, respectively; Fig. 3].

Patient global evaluation $72 \mathrm{~h}$ after the initial dose of parecoxib. Two studies $(15,16)(n=748)$ provided specific data for analysis of PGESM at $72 \mathrm{~h}$ after surgery. We selected the fixed-effect model to perform the meta-analysis since there were no significant heterogeneities (effective, $\chi^{2}=1.71$, $\mathrm{P}=0.19, \mathrm{I}^{2}=41.4 \%$; ineffective $\chi^{2}=1.79, \mathrm{P}=0.18, \mathrm{I}^{2}=44.2 \%$ ). The incidence of 'effective' and 'ineffective' results in the analgesic effect evaluation was significantly different between the two groups [RR=1.30, 95\% CI (1.21-1.40), $\mathrm{P}<0.00001$; and $\mathrm{RR}=0.33,95 \%$ CI (0.23-0.48), $\mathrm{P}<0.00001$, respectively; Fig. 4].

Comparisons of safety. We selected the fixed-effect model to perform the meta-analysis since there were no significant heterogeneities (respiratory depression, $\chi^{2}=0.08, \mathrm{P}=0.77$, $\mathrm{I}^{2}=0 \%$; pruritus, $\chi^{2}=0.05, \mathrm{P}=0.82, \mathrm{I}^{2}=0 \%$; fever, $\chi^{2}=5.39$, $\mathrm{P}=0.25, \mathrm{I}^{2}=25.8 \%$; headache, $\chi^{2}=2.84, \mathrm{P}=0.42, \mathrm{I}^{2}=0 \%$; nausea and vomiting, $\left.\chi^{2}=0.40, \mathrm{P}=0.82, \mathrm{I}^{2}=0 \%\right)$. Two $(15,18)(\mathrm{n}=454)$, two $(14,18)(n=437)$ and four studies $(14-16,19)(n=1171)$ provided data of respiratory depression, pruritus and headache, respectively. The incidence of respiratory depression, pruritus and headache between the treatment and control groups was not significantly different $[\mathrm{RR}=0.84,95 \% \mathrm{CI}(0.38-1.83)$, $\mathrm{P}=0.66 ; \mathrm{RR}=0.91,95 \% \mathrm{CI}(0.54-1.52), \mathrm{P}=0.71$; and $\mathrm{RR}=0.77$, 95\% CI (0.47-1.28), $\mathrm{P}=0.32$, respectively; Fig. 5].

Five $(15-18,20)(n=1048)$ and three studies $(14,18,20)$ $(n=567)$ provided data on fever, and nausea and vomiting, respectively. The incidences of fever, and nausea and vomiting between the treatment and control groups were significantly different $[\mathrm{RR}=0.34,95 \% \mathrm{CI}(0.22-0.53), \mathrm{P}<0.00001$; and $\mathrm{RR}=0.69,95 \%$ CI (0.57-0.83), $\mathrm{P}<0.00001$, respectively; Fig. 5].

\section{Discussion}

NSAIDs are known to induce analgesia mainly via inhibition of COX. Parecoxib exhibits anti-inflammatory, analgesic and antipyretic properties in animal models and humans due to inhibition of prostanoid synthesis primarily by affecting COX-2. Although the inhibition of COX in the periphery is commonly accepted as the primary mechanism, experimental and clinical data suggest a potential role for central COX inhibition to produce antinociception and reduce hypersensitivity. Additionally, it has double analgesic actions (21). Multimodal analgesia, where opioids, including morphine, are administered with a non-opioid, is often used to reduce opioid-related adverse effects, including postoperative nausea and vomiting, drowsiness, respiratory depression and gastrointestinal and bladder dysfunction (22). The underlying principle is that the different modes of action of morphine and the non-opioid drug allow optimum analgesia to be maintained with a lower dose of morphine and consequently a lower incidence of morphine-related adverse effects (23).

We conducted the current meta-analysis to compare the efficacy and safety of parecoxib sodium plus PCA with PCA alone for acute postoperative pain. The results of the metaanalysis indicated that the efficacy of PCA combined with parecoxib sodium (successively injected for $<3$ days intravenously) was superior to that of PCA alone with a statistically significant difference. After 24, 48 and $72 \mathrm{~h}$ of the initial dose of $40 \mathrm{mg}$ parecoxib i.v., the percentage of 'effective' treatment as described by PGESM was higher compared with that of the 


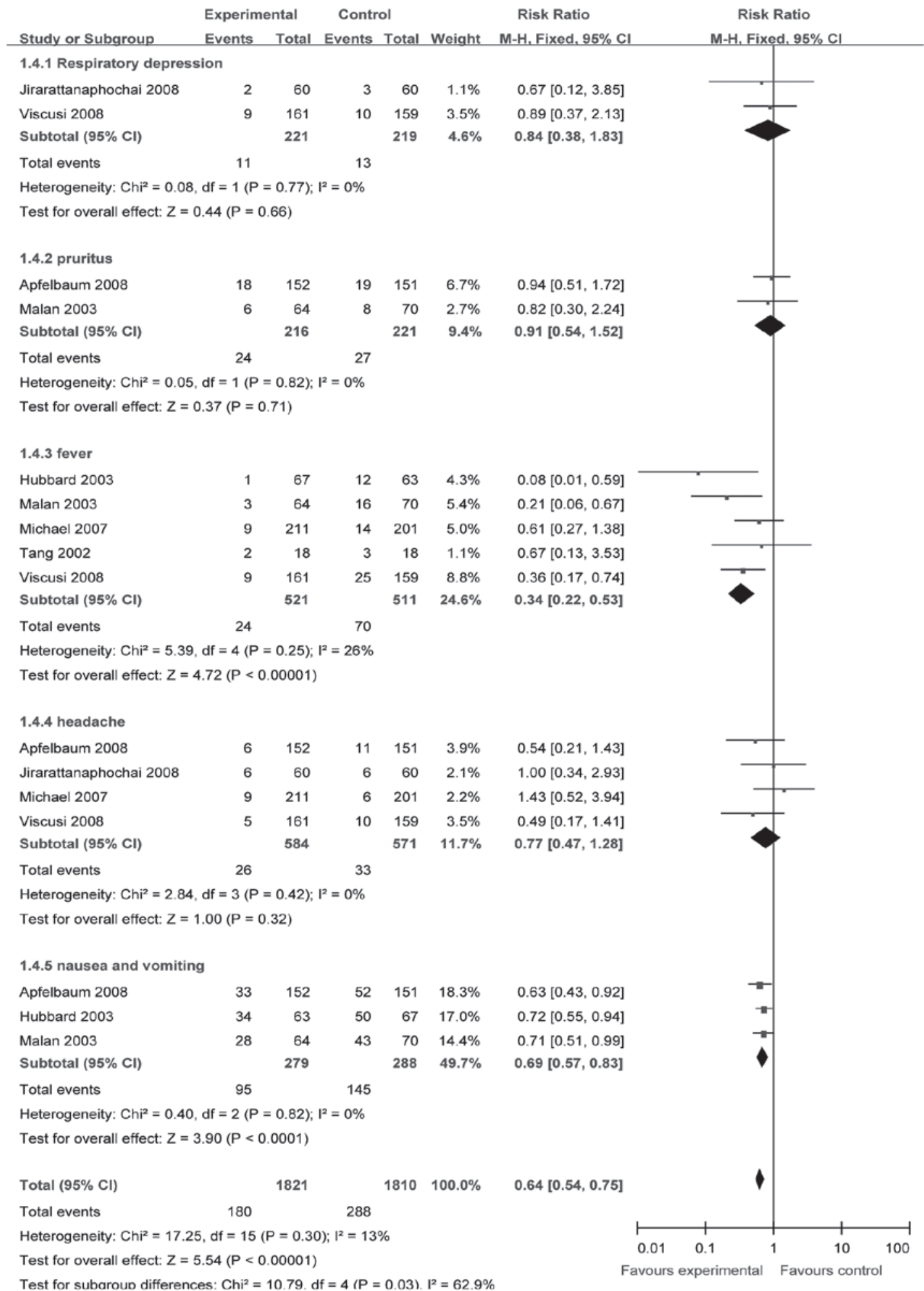

Figure 5. Meta-analysis of adverse drug reactions after surgery of PCA combined with parecoxib sodium and PCA alone. PCA, patient-controlled analgesia.

control group; the percentage of 'ineffective' treatment was lower compared with that of the control group. Moreover, PCA plus parecoxib sodium reduced the incidence of postoperative fever, nausea and vomiting; however, it did not significantly reduce the incidence of respiratory depression, pruritus and headache. The incidence of postoperative bleeding, urinary retention, digestive tract ulcer, pulmonary embolism, massive hemorrhage and cardiovascular events in all the included studies were extremely low, which demonstrated an improved security of parecoxib sodium. However, a previous study indicated that there was a reduction in 24-h morphine consumption, leading to a reduction in morphine-related adverse effects when COX-2 inhibitors were administered in addition to PCA morphine following surgery, with no clear difference between them (23). Therefore, our findings indicate that parecoxib sodium may be beneficial in pain relief 
following surgery; however, further studies are required to confirm this.

Certain limitations affecting the results of the current metaanalysis should be taken into account. Firstly, our findings may be affected by the quality of trials included in the meta-analysis. A well-designed randomized controlled trial requires a thorough understanding of randomization so that better results are achieved. However, none of the included trials clarified allocation concealment clearly. All studies were only assessed to be a Jadad score B in terms of methodology. Secondly, this meta-analysis is based on a relatively small number of RCTs and we acknowledge that using a limited number of studies raises the possibility of a second-order sampling error (24). Thirdly, the distinct differences in administration times, dose, treatment course, different surgery and initial pain level of patients used exist (Table I), which may affect the consistency of effects across those included studies.

In conclusion, although certain limitations exist in this meta-analysis, based on the results of our meta-analysis, we identified that parecoxib is an effective and relatively safe option for acute postoperative pain. However, further high quality $\mathrm{RCTs}$ are required to determine the long-term effects of parecoxib for postoperative pain.

\section{References}

1. Barden J, Derry S, McQuay HJ and Moore RA: Single dose oral ketoprofen and dexketoprofen for acute postoperative pain in adults. Cochrane Database Syst Rev 4: CD007355, 2009.

2. Vane JR: Inhibition of prostaglandin synthesis as a mechanism of action for the aspirin-like drugs. Nat New Biol 231: 232-235, 1971.

3. Needleman P and Isakson PC: The discovery and function of COX-2. J Rheumatol Suppl 49: 6-8, 1997.

4. Rømsing $\mathrm{J}$ and Møiniche S: A systematic review of COX-2 inhibitors compared with traditional NSAIDs, or different COX-2 inhibitors for post-operative pain. Acta Anaesthesiol Scand 48: 525-546, 2004

5. Lloyd R, Derry S, Moore RA and McQuay HJ: Intravenous or intramuscular parecoxib for acute postoperative pain in adults. Cochrane Database Syst Rev 2: CD004771, 2009.

6. Graff J, Arabmotlagh M, Cheung R, Geisslinger G and Harder S: Effects of parecoxib and dipyrone on platelet aggregation in patients undergoing meniscectomy: a double-blind, randomized, parallel-group study. Clin Ther 29: 438-447, 2007.

7. Harris SI, Stoltz RR, LeComte D and Hubbard RC: Parecoxib sodium demonstrates gastrointestinal safety comparable to placebo in healthy subjects. J Clin Gastroenterol 38: 575-580, 2004.

8. Noveck RJ, Laurent A, Kuss M, Talwalker S and Hubbard RC: Parecoxib sodium does not impair platelet function in healthy elderly and non-elderly individuals: two randomised, controlled trials. Clin Drug Investig 21: 465-476, 2001.
9. Stoltz RR,Harris SI, Kuss ME,LeComteD, Talwalker S, Dhadda S and Hubbard RC: Upper GI mucosal effects of parecoxib sodium in healthy elderly subjects. Am J Gastroenterol 97: 65-71, 2002.

10. Sackett DL, Clarke M, Oxman AD: Cochrane Reviewers, Handbook 4.2. In: Renew Manager. Versions 4.2. Oxford, England. The cochrane collaboration, 13-36, 2002.

11. Jüni P, Altman DG and Egger M: Systematic reviews in health care: Assessing the quality of controlled clinical trials. BMJ 323: 42-46, 2001.

12. Jadad AR, Moore RA, Carroll D, Jenkinson C, Reynolds DJ, Gavaghan DJ and McQuay HJ: Assessing the quality of reports of randomized clinical trials: is blinding necessary? Control Clin Trials 17: 1-12, 1996.

13. Jüni P and Egger M: PRISMAtic reporting of systematic reviews and meta-analyses. Lancet 374: 1221-1223, 2009.

14. Apfelbaum JL, Desjardins PJ, Brown MT and Verburg KM: Multiple-day efficacy of parecoxib sodium treatment in postoperative bunionectomy pain. Clin J Pain 24: 784-792, 2008.

15. Michael C, Artur J, Kotarski J, Katz TK, Brown MT and Verburg KM: Parecoxib sodium administered over several days reduces pain after gynecologic surgery via laparotomy. J Clin Anesth 19: 448-455, 2007

16. Viscusi ER, Gimbel JS, Halder AM, Snabes M, Brown MT and Verburg KM: A multiple-day regimen of parecoxib sodium $20 \mathrm{mg}$ twice daily provides pain relief after total hip arthroplasty. Anesth Analg 107: 652-660, 2008.

17. Tang J,Li S, White PF, Chen X, Wender RH, Quon R, Sloninsky A, Naruse R, Kariger R, Webb T and Norel E: Effect of parecoxib, a novel intravenous cyclooxygenase type-2 inhibitor, on the postoperative opioid requirement and quality of pain control. Anesthesiology 96: 1305-1309, 2002.

18. Malan TP Jr, Marsh G, Hakki SI, Grossman E, Traylor L and Hubbard RC: Parecoxib sodium, a parenteral cyclooxygenase-selective inhibitor, improves morphine analgesia and is opioid-sparing following total hip arthroplasty. Anesthesiology 98: 950-957, 2003.

19. Jirarattanaphochai K, Thienthong S, Sriraj W, Jung S, Pulnitiporn A, Lertsinudom S and Foocharoen T: Effect of parecoxib on postoperative pain after lumbar spine surgery. Spine 33: 132-139, 2008.

20. Hubbard RC, Naumann TM, Traylor L and Dhadda S: Parecoxib sodium has opioid-sparing effects in patients undergoing total knee arthroplasty under spinal anaesthesia. Br J Anaesth 90: $166-172,2003$

21. Koppert W, Wehrfritz A, Körber N, Sittl R, Albrecht S, Schüttler J and Schmelz M: The cyclooxygenase isozyme inhibitors parecoxib and paracetamol reduce central hyperalgesia in humans. Pain 108: 148-153, 2004.

22. Beaulieu P: Non-opioid strategies for acute pain management. Can J Anaesth 54: 481-485, 2007.

23. Maund E, McDaid C, Rice S, Wright K, Jenkins B and Woolacott N: Paracetamol and selective and non-selective non-steroidal anti-inflammatory drugs for the reduction in morphine-related side-effects after major surgery: a systematic review. Br J Anaesth 106: 292-297, 2011.

24. Higgins J, Thompson S, Deeks J and Altman D: Statistical heterogeneity in systematic reviews of clinical trials: a critical appraisal of guidelines and practice. J Health Serv Res Policy 7: 51-61, 2002. 